\title{
Vascular Shape Segmentation and Structure Extraction Using a Shape-Based Region-Growing Model
}

\author{
Yoshitaka Masutani, Thomas Schiemann and Karl-Heinz Höhne \\ Institute of Mathematics and Computer Science in Medicine (IMDM), \\ University Hospital Hamburg - Eppendorf \\ Martinistr. 52, 20246 Hamburg, Germany \\ Tel. +49-40-4717-3652, Fax. +49-40-4717-4882 \\ masutani@uke.uni-hamburg.de
}

\begin{abstract}
A new, practical, and efficient approach is proposed for 3D vascular segmentation and bifurcation structure extraction. The method uses a combination of mathematical morphology, region-growing schemes, and shape features in addition to greyscale information. By an extension of math-morphological operations within bounded space of vascular shape, smooth and natural region-growing and sensitivity-controllable bifurcation detection were realized. The algorithm was implemented in the interactive segmentation and visualization software package VOXELMAN and validated with clinical data of X-ray CT angiography and MRA.
\end{abstract}

\section{Introduction}

Segmentation is one of the most important and difficult procedures in medical image analysis and in its clinical application, and blood vessels are especially difficult to segment. Except for large structures like the abdominal aorta, even visualization is not as easy as for other organs. Additional structural information is often required for special purposes beyond visualization and segmentation, for example construction of a structured vascular atlas [Pommert94], functional analysis of blood supply [Hoehne95], or navigation of catheters for endovascular treatment [Masutani97]. These procedures need information like bifurcation structure or radii of blood vessels. As seen in several reports, common methods for obtaining such information are usually based on three-dimensional thinning algorithms [Tsao81][Malandain93][Szekely94]. However, these thinning-based approaches fail to manage abnormal vessel structures like aneurysms which are important in clinical routine. Preliminary separation of such objects from vessels [Masutani95] is suitable to measure their volumes, however the relationship between them (e.g. neck shape of aneurysm) is spoiled.

The most serious problem in thinning is that such algorithms try to keep topology of original objects. Therefore, segmentation procedures must be carefully carried out to get topologically correct vascular shapes. However, practically, this is too hard to achieve without any manual correction or compilation. The main reason for such difficulties is low resolution of current medical imaging modalities regarding the size of blood vessels. This causes partial volume effects which connect vascular structures to other organs like bones in X-ray CT. In case of MRA (Magnetic Resonance Angiography), flow-void, which is lack of signals in vessels, often causes disconnection of extracted vascular objects. Therefore, it is almost impossible to solve these problems without any a priori knowledge. For such reasons, recent methods using deformable models are promising for relatively larger structures like brains or livers. Because these methods originally assume and utilize global smoothness of the target objects. However, blood vessels are not globally smooth and have bifurcation structures. Recent reports by McInerney [McInerny97] showed an improvement for such drawback by a more flexible deformable model.

Our goal is to obtain structured vascular shape with minimum interaction for constructing a digital atlas, image-guided surgery, and so on. For these purposes, vascular structures must be separated in groups of vessel branches and abnormal structures. Therefore, we developed a region (voxel)-based approach for the convenience of bifurcation analysis. In this paper, we propose a new model based on region-growing controlled by math-morphological information of local shape, which has the ability of topological correction. 


\section{Materials and Methods}

1. Overview

Our method for vascular shape segmentation and structure extraction consists of two parts. First, the initial shape is acquired by thresholding. Then, region-growing is processed basically in the space limited by the initial shape. Structural information of the shape is simultancously obtained. The region grows while avoiding nonvessel regions and keeps its local smoothness based on math-morphological information and local shape processing. In the next two sections, details of mathmorphological information and local mathmorphological shape processing are described.

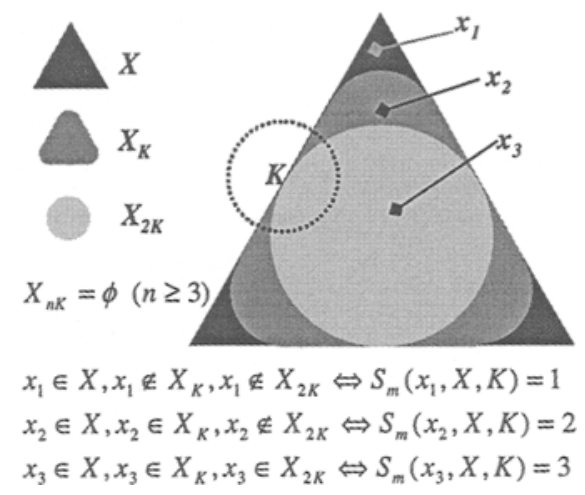

Fig. 1. Computation example of morphological size

\section{Mathematical morphology}

Mathematical morphology is a well-known theory for structure analysis and processing of binary shapes [Robert87]. Most math-morphological operations and analysis methods can be defined in terms of the basic operations erosion, dilation, closing and opening. One of the most important characteristics of them is controllability of their results by size and shape of a filter kernel (structuring element). A math-morphological quantity, called morphological size, is used in our region-growing model. It is an attribute of voxels which belong to an object. Distribution of morphological size in a shape is called pattern spectrum [Maragos89] to represent the characteristics of the whole shape.

Let $x$ denote a voxel in a shape $X, K$ a kernel for opening and $n K$, the $n$-scaled kernel of $K$. Morphological size of a voxel $x, S_{m}(x, X, K)$ is defined as:

$$
S_{m}(x, X, K)=\min \left\{n \mid x \in X, x \notin X_{n K}\right\} \quad(n \geq 1)
$$

where $X_{K}$ reperesent the shape of $X$ opened by $K$. That is, morphoiogical size of a voxel in an object means the minimum size of kernels which can remove the voxel from the shape by opening operation (Fig.1). It represents local thickness of the object at the position of voxel $x$.

\section{Mathematical morphology in bounded space}

Math-morphological operations are usually performed in unlimited space. In this paper, an extension of these operations in bounded space is introduced to generalize region growth and to process the shape of its growth-front. Beside $X$ and $K$, give a constant boundary shape $B$ which is never changed by any operations and let $B^{C}$ be its complement. The two most fundamental operations, dilation and erosion are defined as follows:

\section{Bounded space dilation of a shape $X$ in $B$ by $K$ :}

$$
\begin{aligned}
& X \bullet_{B} K=X_{N}=\left(X_{N-1} \bullet \tilde{K}\right) \cap B=\ldots \quad \bullet: \text { original dilation } \\
& X_{i}=\left(X_{i-1} \bullet \tilde{K}\right) \cap B, \quad X_{0}=X, \widetilde{K}=\frac{1}{N} K
\end{aligned}
$$

\section{Bounded space erosion of a shape $X$ in $B$ by $K$ :}

$$
X \circ_{B} K=\left(\left(X^{c} \cap B\right) \bullet_{B} K\right)^{c} \cap B
$$

$N$ must be large enough to avoid jump and diffraction of growing as shown in Fig.2. In discrete space, the radius of $\tilde{K}$ is 1 .

Most properties of original dilation and erosion, such as translation invariability, distributivity, local knowledge, and increasing are kept in bounded space extension while iterativity is not. Regarding the definition of bounded space dilation, our previous approach [Masutani96] 
prohibited only jump of operations using a connectivity filter. This new method also restricts diffraction of growing.

Like original closing and opening, bounded space versions of closing and opening are defined by combinations of the above operations:

Bounded space closing of a shape $X$ in $B$ by $K$ :

$$
{ }^{B} X^{K}=\left(X \cdot{ }_{B} K\right){ }_{B} K
$$

Bounded space opening of a shape $X$ in $B$ by $K$ :

$$
{ }^{B} X_{K}=\left(X{ }_{B} K\right) \bullet_{B} K
$$

They also have smoothing effects on shapes. Two interesting properties for smoothing are convex-filling (closing only) and growth-front smoothing as shown in Fig.3. Zahlten proposed a method [Zahlten95] similar to our model and reported that a growth-front sometimes becomes oblique to its growing direction after going through narrow and curved parts of bounded space. Bounded space closing or opening with a kernel of relatively large size tries to modify such an oblique front perpendicular to the growing direction. As shown in Fig.3(a), the kernel radius must be larger than the width of the narrow structure to get obvious smoothing effect. The convex-filling property shown in Fig.3(b) is important to control the sensitivity of the bifurcation detection. This property is explained in the next section with a simplified example.

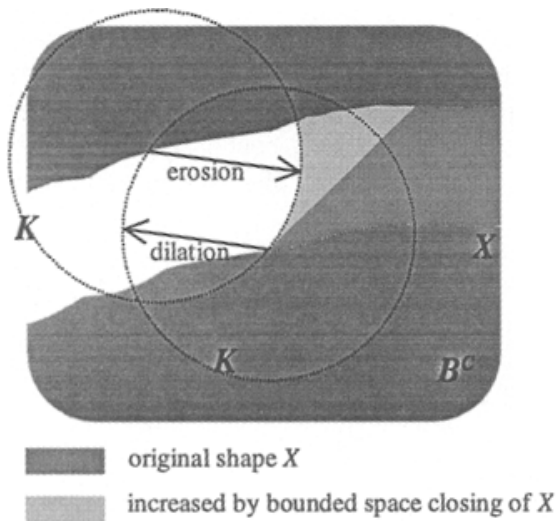

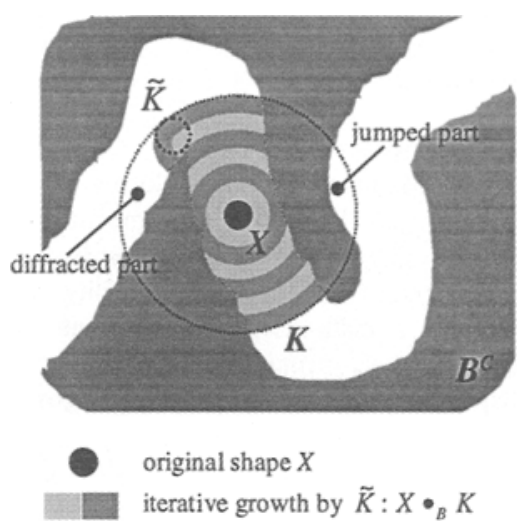

Fig. 2. Bounded space dilation $(N=6)$

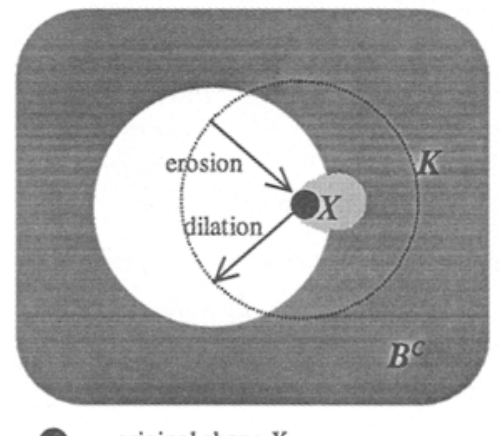

original shape $X$

filled by bounded space closing of $X$

Fig. 3. Properties of bounded space closing. Growth-front smoothing (left) and convex filling (right)

\section{Description of the algorithm}

\section{(a) Initial shape acquisition}

A thresholding operation is performed to get an initial binary shape. Except for blood vessels, it may include other structures like bone, and some vessels may be connected to other vessels, or may 
be partially disconnected (Fig.4). Threshold values (minimum and maximum) should be interactively adjusted to minimize the need for correction by the following processing.

\section{(b) Seed definition}

A seed is positioned, which defines the starting shape for iterative dilation in bounded space.

(c) Shape-based region-growing model

The model iterates basically two processes, simple growing and growth-front smoothing. The former is bounded space dilation and the latter is bounded space closing, both with different kernels. Other optional processes and growing conditions for

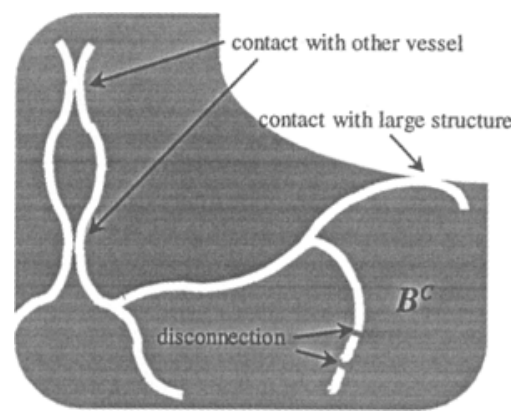

Fig. 4. Example of initial shape certain purposes are defined in section (d).

A compact set of voxels, which belong to the same growth generation, is defined as a cluster, which is an unit of iterative growth (Fig.5). $C_{0}$ denotes a seed voxel (or region), and $C_{1}$ is a differential regions after the first growth step. If voxels after bounded space dilation are disconnected, these regions must be distinguished as individual clusters. This situation defines bifurcation. Therefore, for an initial boundary shape $B$, clusters grow as:

$$
\bigcup_{i=0}^{m} C_{n+i}=\left\{\left(\bigcup_{i=0}^{n-1} C_{l}\right) \bullet_{B} K_{G}\right\}^{K_{s}}(n \geq 1, m \geq 0)
$$

where $m$ is summation of bifurcation, $K_{G}$ and $K_{S}$ are kernels for simple-growing and growth-front smoothing respectively.

During iterative cluster generation, attributes of clusters are measured (e.g. volume, growing direction, center of gravity, morphological size and connectivity to other clusters).

\section{(d) Optional growing configuration}

\section{-morphological size upper limitation}

We used the morphological size computed with a spherical kernel radius 1 as an attribute similar to greyscale values. Similar to conventional regiongrowing, morphological size is used for limitation of the growing process. Note that this condition is not applied in growth-front smoothing. This operation prevents clusters from explosive growing into connected large structures.

\section{-object boundary opening of clusters}

This operation is used to neglect thin contacts of a cluster with other structures (Fig.6). It is realized by conventional opening. However, erosion and dilation must be from/to object boundary not from/to cluster boundary. Therefore, a cluster $C$ processed by object boundary opening $C_{K}$ is:

$$
C_{K}=C \cap B_{K}
$$

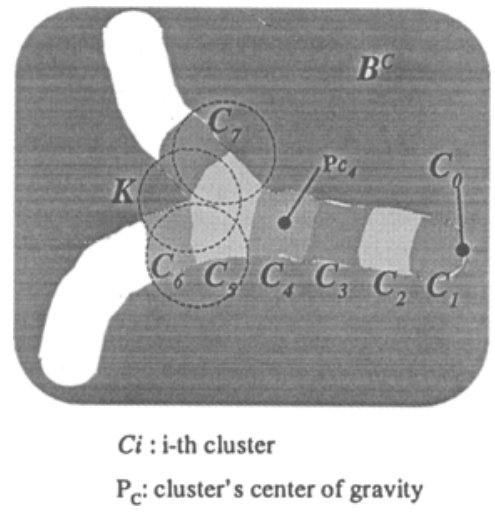

Fig. 5. Shape-based region-growing model

where $B$ is a boundary shape and $K$ is a kernel.

\section{-directional growing}

The kernel shape for simple growing $K$ should be spherical so that it can grow in any direction 
uniformly. However, if a kernel is directional and its direction is controlled by the growing dircction, growing skips several bifurcation structures (Fig.7). A directional version of $K$ in a direction $v, K_{v}$ is:

$$
K_{v}(t)=\left\{y \mid y \in K, \frac{y}{|y|} \cdot \frac{v}{|v|} \geq t\right\} \quad(-1 \leq t \leq 1)
$$

where $t$ is a threshold value for the dot product and therefore $K_{v}(-1,0)=K$.

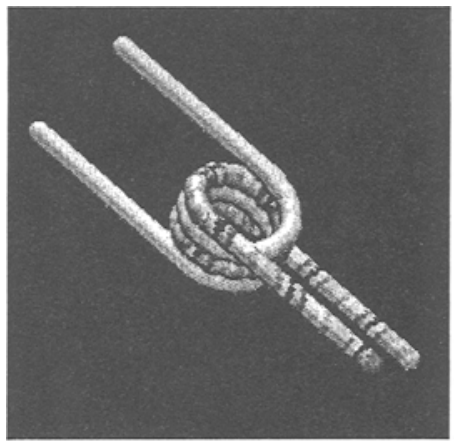

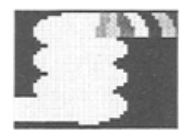

(1)

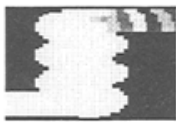

(3)

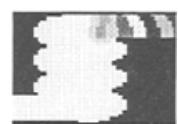

(2)

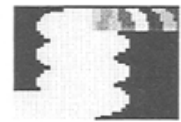

(4)

Fig. 6. Neglecting thin contact by cluster opening (synthetic data). The whole shape and final result are shown (left). Clusters are painted in repeated 12 gray tones. The procedure of cluster opening is shown (right). From a cluster (1), the region grows over thin contact (2). By erosion of the cluster and the region, the contact is removed and the disconnected region is removed (3). Dilation recovers the thickness of the clusters (4).

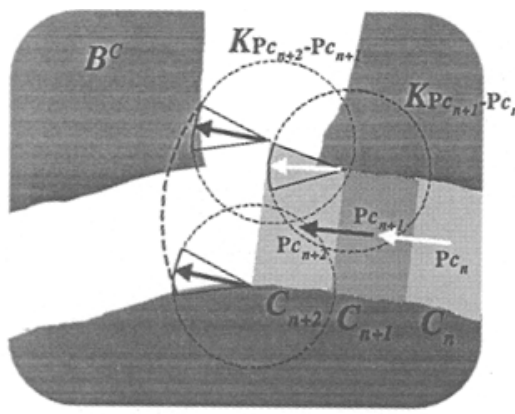

$\mathrm{Ci}: \mathrm{i}$-th cluster
$P_{c}$ : cluster's center of gravity

Fig. 7. Directional growing

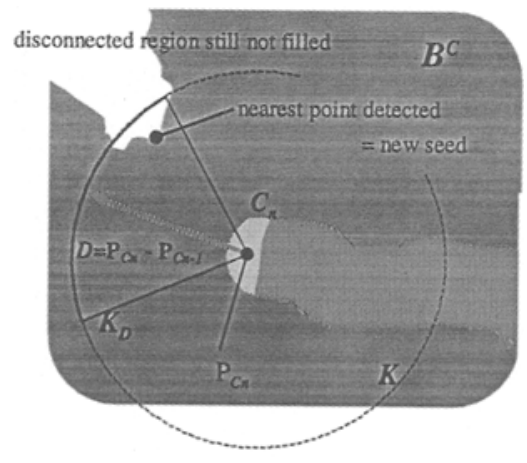

Fig. 8. Directional jump growing from an end cluster

\section{-directional jump growing}

If no region grows after several iterations, clusters with connectivity number 1 try to grow out of the initial shape. This means that local ends try to establish connections to other regions (Fig.8), which have not been filled before. These ends search disconnected regions with a directional kernel. If such regions are detected, a new seed is thrown to continue further growing. This process is iterated until no disconnected region is detected.

\section{(e) Control of kernel sizes and shapes}

Basically, kernel shapes are spherical both for simple growing and growth-front smoothing. Since the size of the growing kernel $K_{G}$ controls the length of the clusters in their growth directions, the kernel radius should be 1 . Otherwise, in the final process described in section (f), certain quantization effects of branch grouping are observed. However, for the kernels with radius 1 of digital spherical shape, directional control is difficult. Therefore, directional kernels should have relatively 
larger radius (about 5 voxels). The radius of the growth-front smoothing kernel controls the sensitivity of the bifurcation detection. With smaller kernels, smaller convex shapes are detected as bifurcation. As shown in Fig.8(a), a convex part of a vascular shape is detected as bifurcation, if a growth-front smoothing kernel does not completely fill the convex structure in the bounded space dilation process when the growth-front reached the boundary to separate the rest of the shape into two parts. While this bifurcation detection property depends on the width $W$ and the length $L$ of the convex shape, it is obvious and interesting that it is independent of the local width $D$ of the main branch, if the kernel size is constant.

In our model, the radius of the kernel is adaptively set as the width of the previous cluster in order to smooth the growth-front. The cluster width is defined as double of its morphological size. Therefore, convex shapes with width 1 and length smaller than $D$ are not detected as bifurcations. Fig.8(b) shows examples of neglected convex shapes.

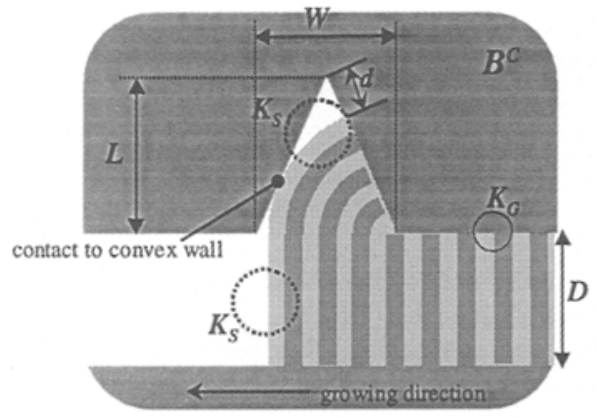

If $d>$ radius of $K s$, bifurcation is detected.

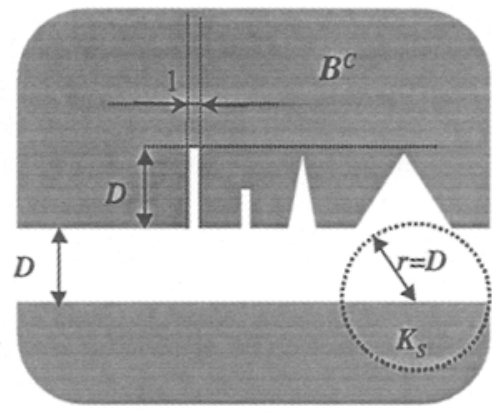

Fig. 8. Bifurcation detection control. (a) an example of bifurcation detection (left) (b) examples of neglected convex shapes in the case of $r=D$ (right)

\section{(f) Cluster grouping (Constructing branches)}

Finally, a set of clusters with their connection graph is obtained as an extracted shape. To make anatomically relevant information, i.e. sets of branches or other structures, clusters are grouped based on their attributes. Making branches is realized by grouping of clusters according to their connectivity number. A branch is defined as a set of connected clusters, which have connectivity numbers smaller than 3 . Those branches can be further divided into several groups according to their growing directions. Other connected clusters with irregularly larger volumes are also grouped, e.g. abnormal structures like aneurysms.

\section{Results}

This algorithm was implemented within the segmentation section [Schiemann92] of the software VOXEL-MAN [Hoehne95]. Except for initial shape acquisition and seed definition, users select one of the modes for optional processing described above. The growth termination condition is also variable. For example, if bifurcation detection is set as condition, it functions as a single branch grabber tool. Other conditions are limitation of total volume, cluster volume or generation of growth.

Interactive segmentation of cerebral blood vessel structures was performed on clinical data

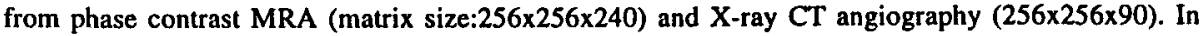
Fig.10, an initial shape was obtained from MRA, and clusters were grouped into branches in different colors (12 colors repeated). For X-ray CT data, a vessel, which contacted with the skull bone was extracted using limitation of morphological size between 1 and 3 (Fig.11).

\section{Discussion and Summary}

Based on the extension of mathematical morphology to bounded space, a new model for segmentation and structure extraction of vascular shapes was proposed. By theoretical investigation 
and clinical application, new features of the model, like quantitative bifurcation detection, its sensitivity control and topological correction of vascular shapes were shown.

As it is often discussed, conventional thinning algorithms yield noisy branches due to high sensitivity to convex shape. One of the important properties of our model is controllability of the bifurcation detection by the size of the growth-front smoothing kernel. The definition of sensitivity for bifurcation gave reasonable results for cerebral vascular shapes.

In our results, any line structure enhancement filters were not employed. Such filters may contribute to a reduction of disconnection in the initial shape. As shown in detail by Sato [Sato98], however, false responses like connection between adjacent vessels spoil small structures. We also investigated response properties for other situations like contact with bone and non-vessel like structures (c.g. ancurysms) to conclude that more improvement is needed for our purpose.

In cerebral arteries, some vessels are less than three voxels in diameter. This is critical for use of object boundary opening to avoid thin contact with other vessels. For such cases, directional kernels showed better performance. The behavior of our model, especially with optional growing conditions can be compared to a fluid with a certain viscosity. Use of parameters in such analogy may give a better user-interface than the kernel radius or the threshold for dot products.

Future work will address shape interpolation between end clusters and disconnected regions. For non-circular sections of vessels (e.g. sagittal sinus), shapes of end clusters should be utilized.

\section{Acknowledgement}

The authors are grateful to all the members in IMDM, who developed the superior visualization software package VOXEL-MAN and guest researcher Dr. Akinobu Shimizu from Nagoya University, Japan for giving practical comments and advice. This project is partially supported by DAAD (German Academic Exchange Service).

\section{References}

[Pommert94] A. Pommert, et al. Symbolic Modeling of Human Anatomy for Visualization and Simulation, SPIE Vol. 2359, Proc. of Visualization on Biomedical Computing (VBC) 94, pp412-423, 1994

[Hoehne95] K. H. Höhne, et al. A new representation of knowledge concerning human anatomy and function, Nature Medicine, Vol.1, No. 6, 1995

[Masutani97] Y. Masutani, et al. Interactive Virtualized Display System for Intravascular Neurosurgery, LNCS Vol. 1205, Proc. of CVRMed (Computer Vision Virtual Reality and Robotics in Medicine) '97, pp427-434, 1997

[McInerney97] T. Mclnerney, et al. Medical image segmentation using topologically adaptable surfaces, LNCS Vol. 1205, Proc. of CVRMed'97, pp23-32, 1997

[Szckely94] G. Szekely, et al. Structural description and combined 3-D display for superior analysis of cerebral vascularity from MRA, SPIE Vol. 2359, Proc. VBC'94, pp272-281, 1994

[Tsa081] Y. F. Tsao, et al. A Parallel Thinning Algorithm for 3-D Pictures, Computer Graphics and Image Processing Vol.17, 1981 pp315-331

[Malandain93] G. Malandain, et al. Topological segmentation of discrete surfaces, International Journal of Computer Vision, Vol.10, No.2, pp 183-197, 1993

[Masutani95] Y. Masutani, et al. Quantitative Vascular Shape Analysis for 3D MR-Angiography Using Mathematical Morphology, LNCS Vol. 905, Proc. CVRMed '95, pp449-454, 1995

[Robert87] M. Robert, et al. Image Analysis Using Mathematical Morphology, IEEE trans. Pattern Analysis and Machine Intelligence, Vol. PAMI-9, No. 4, pp532-550, 1987

[Maragos89] P. Maragos, et al. Pattern Spectrum and Multiscale Shape Representation, IEEE trans. Pattern Analysis and Machine Intelligence, Vol. PAMI-11, No. 9, pp701-716, 1989

[Masutani96] Y. Masutani, et al. Region-Growing Based Feature Extraction Algorithm for Tree-like Objects, LNCS Vol. 1131, Proc. VBC '96, pp161-171, 1996

[Zahlten95] C. Zahlten, et al. Reconstruction of branching blood vessels from CT-data, Proc. 5th Eurographics Workshop on Visualization in Scientific Computing, pp41-52, 1995

[Schiemann92] T. Schiemann, et al. Interactive 3D-segmentation, SPIE Vol. 1808, Proc. VBC '92, pp376-383, 1992

[Sat098] Y. Sato, et al. Three-dimensional multi-scale line filter for segmentation and visualization of curvilinear structures in medical images, Medical Image Analysis vol.2, No.2, pp143-168, 1998 

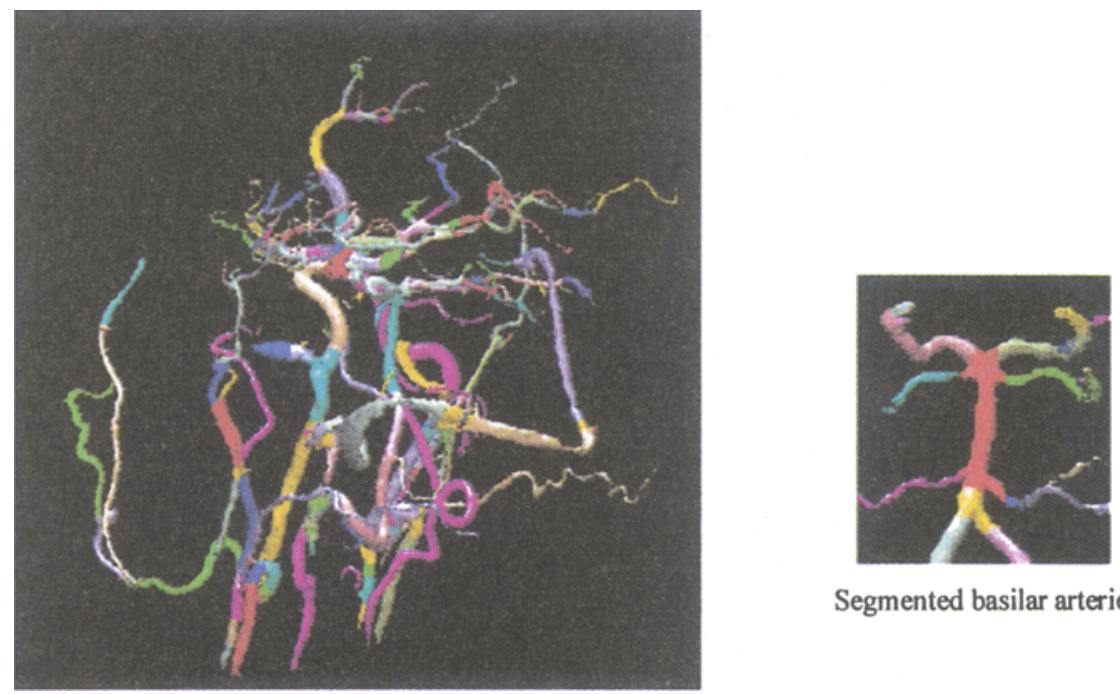

Segmented basilar arteries

Fig. 10. Clinical application results (1), Structures of cerebral arteries (MRA). After defining a seed, the whole object was structured as clusters and these were grouped into branches using the connectivity number (left: Branches are colored in repeated 12 colors). A part of the whole structure, basilar arteries are shown (right).
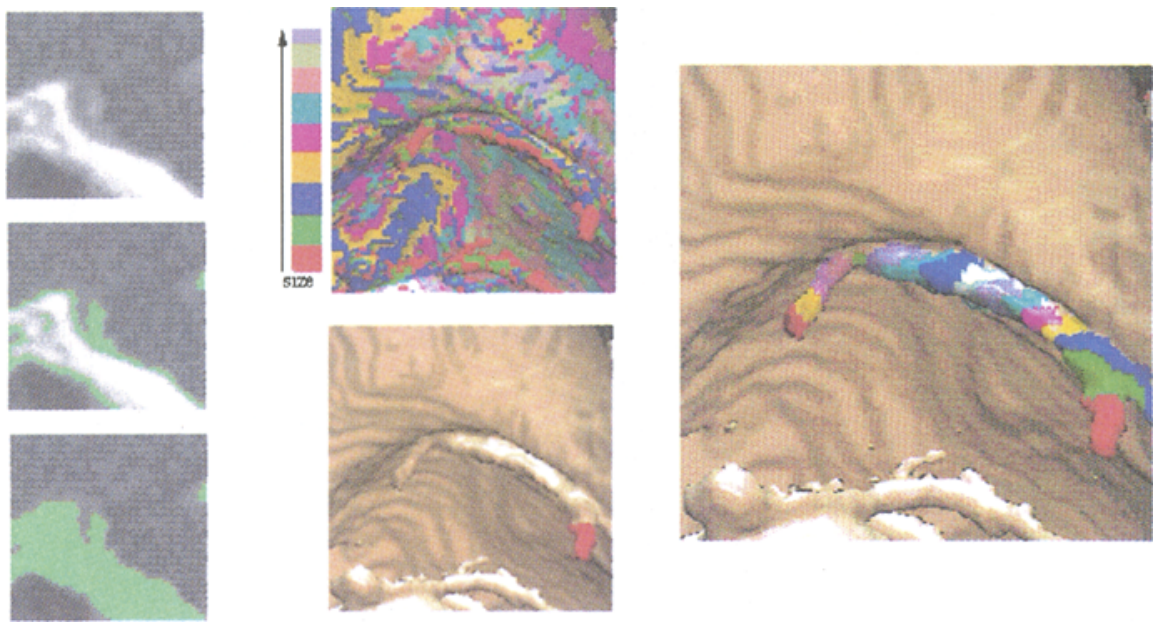

Fig. 11. Clinical application results (2), Bone structure avoidance (X-ray CT angiography). Adjustment of threshold values can not separate bone and vessels because of the partial volume effects (left). Using the threshold range shown in the bottom image (left), the morphological size was computed (middle upper). A seed area is given on the vessel (middle lower), the vessel was extracted (right, Clusters are shown in repeated 12 colors). 